MS40. X-ray diffraction from microsecond to femtosecond time range (including FELs)

Chairs: Christian Betzel, Anton Barty

\section{MS40-P1 Time-resolved X-ray diffraction study of inhomogeneous deformations in piezoelectric single crystals, induced by a nanosecond electric pulse}

Hyeokmin Choe $^{1}$, Semën Gorfman ${ }^{1}$, Michael Ziolkowski ${ }^{1}$, Marco Vogt $^{1}$, Stefan Heidbrink ${ }^{1}$, Ullrich Pietsch ${ }^{1}$

1. Department of Physics, University of Siegen, Walter-Flex Str. 3, D-57072 Siegen, Germany

email: choe@physik.uni-siegen.de

Electromechanical coupling (piezoelectric effect) is the physical property describing the ability of some materials to convert electrical energy to mechanical energy or vice versa. Despite the great technological importance of piezoelectric materials, many fundamental aspects of piezoelectricity are still quite poorly understood. For instance, very little are known about a reaction of piezoelectric crystals to ultra-short (e.g. nanosecond) and strong electric pulses on the microscopic and mesoscopic length scales. The aim of this work is to investigate the reaction of lithium niobate $\left(\mathrm{LiNbO}_{3}\right)$ and $\alpha$-quartz $\left(\alpha-\mathrm{SiO}_{2}\right)$ single crystals to a nanosecond electric pulse by time-resolved X-ray diffraction.

We report on the measurement of time-dependent rocking curves of selected Bragg reflections from the $\sim 0.05 \mathrm{~mm}$ thin single crystal plates. We applied $2 \mathrm{~ns}$ electric pulse reaching the maximum amplitude of $30 \mathrm{kV} / \mathrm{mm}$ and collected diffraction intensity as a function of time delay after the applied pulse. The measurement was performed using the specially developed stroboscopic data-acquisition system [1-3]. Figure1 shows dynamics of $0 \quad 0 \quad 12$ Bragg rocking curve of $\mathrm{LiNbO}_{3}$ as a function of time (passed after the pulse). We observed the range of phenomena, corresponding to the changes on different time and length scales, including: (1) the change of integrated intensity, and (2) change of full width half maxima. The change of integrated intensity can be caused by both, the rearrangement within the unit cell (change of the structure factors) or alternatively by the change induced of the mosaicity (change of the extinction parameter).

[1] S. Gorfman, O. Schmidt, M. Ziolkowski, M. Kozierowski, and U. Pietsch, J. Appl. Phys. 108, 064911 (2010)

[2] S. Gorfman, O. Schmidt, V. Tsirelson, M. Ziolkowski, and U. Pietsch, Z. Anorg. Allgem. Chem. 639(11), 1953-1962 (2013)
[3] S. Gorfman, Crystallogr. Rev. 20(3), 210-232 (2014)

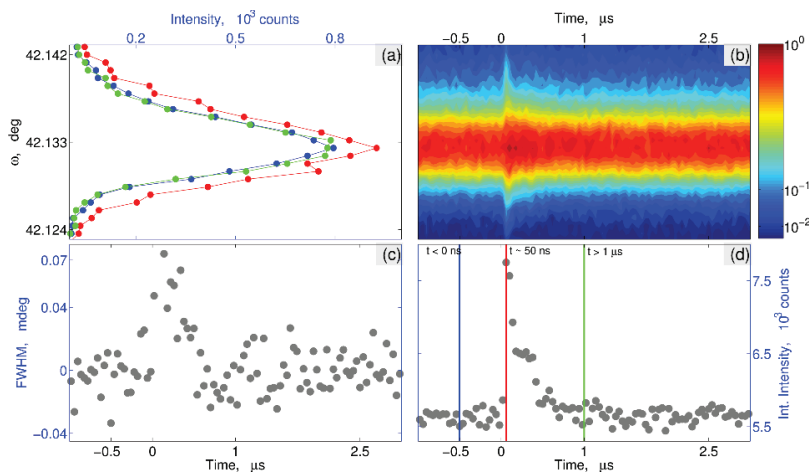

Figure 1. Time-resolved X-ray diffraction of LiNbO single crystal, impacted by nanosecond high electric pulse: (a) dynamics of 0012 Bragg rocking curves as selected time ranges, (b) time-resolved intensity map, (c) change of peak width, and (d) change of integrated intensity after applied electric pulse

Keywords: time-resolved X-ray diffraction, piezoelectric materials, nanosecond electric pulse 